\title{
Evaluation of a C57BL/6J x 129S1/SvImJ Hybrid Nestin-Thymidine Kinase Transgenic Mouse Model for Studying the Functional Significance of Exercise-Induced Adult Hippocampal Neurogenesis
}

\author{
G.F. Hamilton*, P. Majdak, D.S. Miller, P.J. Bucko, J.R. Merritt, C.P. Krebs and J.S. Rhodes \\ Department of Psychology, The Beckman Institute, 405N Mathews Ave, University of Illinois \\ at Urbana-Champaign, Urbana, IL, USA
}

\begin{abstract}
New neurons are continuously generated in the adult hippocampus but their function remains a mystery. The nestin thymidine kinase (nestin-TK) transgenic method has been used for selective and conditional reduction of neurogenesis for the purpose of testing the functional significance of new neurons in learning, memory and motor performance. Here we explored the nestin-TK model on a hybrid genetic background (to increase heterozygosity, and "hybrid vigor"). Transgenic C57BL/6J (B6) were crossed with 129S1/SvImJ (129) producing hybrid offspring (F1) with the B6 half of the genome carrying a herpes simplex virus thymidine kinase (TK) transgene regulated by a modified nestin promoter. In the presence of exogenously administered valganciclovir, new neurons expressing TK undergo apoptosis. Female B6 nestin-TK mice $(n=80)$ were evaluated for neurogenesis reduction as a positive control. Male and female F1 nestin-TK mice $(n=223)$ were used to determine the impact of neurogenesis reduction on the Morris water maze (MWM) and rotarod. All mice received BrdU injections to label dividing cells and either valganciclovir or control chow, with or without a running wheel for 30 days. Both the F1 and B6 background displayed approximately $50 \%$ reduction in neurogenesis, a difference that did not impair learning and memory on the MWM or rotarod performance. Running enhanced neurogenesis and performance on the rotarod but not MWM suggesting the F1 background may not be suitable for studying pro-cognitive effects of exercise on MWM. Greater reduction of neurogenesis may be required to observe behavioral impacts. Alternatively, new neurons may not play a critical role in learning, or compensatory mechanisms in pre-existing neurons could have masked the deficits. Further work using these and other models for selectively reducing neurogenesis are needed to establish the functional significance of adult hippocampal neurogenesis in behavior.
\end{abstract}

Keywords: Running, morris water maze, learning, memory, BrdU, rotarod

\section{INTRODUCTION}

In many mammals including humans and rodents, new neurons are continuously generated in the subgranular zone (SGZ) of the hippocampal dentate gyrus [1-5]. The new cells integrate into the circuitry and

*Correspondence to: Dr. Gillian Hamilton, 3315 Beckman Institute, 405 North Mathews Avenue, Urbana, Illinois 61801, USA. Tel.: +1 217300 5747; E-mail: gillianh@illinois.edu. ultimately behave as normal mature granule neurons [6-9]. However, when they are young, they possess unique morphological and electrophysiological properties, including a depolarizing response to GABA neurotransmission as well as decreased threshold for displaying long term potentiation (LTP), suggesting that new neurons may display greater plasticity, or ability to mold to new experiences than older neurons $[7,10,11]$. 
The processes of neurogenic proliferation, differentiation and survival are highly sensitive to trophic factors among many other chemical features of the microenvironment within the SGZ [12], which in turn are modulated by intrinsic and extrinsic factors such as stress, diet, drugs, genetics and physical activity [13-19]. In particular, aerobic exercise, in the form of voluntary wheel running, has demonstrated robust enhancement of neurogenesis in the hippocampus and increased performance on certain memory tasks in rodent models [20, 21]. In humans, physical activity is also associated with enhanced cognitive performance [22]. Still, while aerobic exercise has been correlated with increased levels of neurogenesis and spatial memory improvement in rodents, causation is difficult to ascertain.

In fact, multiple attempts have been made to discern the causal relationship between levels of hippocampal adult neurogenesis and behavioral performance. Through the use of multiple models and methods, such as focal gamma irradiation, systemic administration of anti-mitotic agents (such as methylazoxymethanol) and genetically engineered mice featuring inducible ablation, a causal relationship between hippocampal adult neurogenesis and behavior has been implied [23-27]. However, more recently, the Flint group published a meta-analysis of all published studies examining the functional role of adult neurogenesis and found no significant effects of ablation of adult neurogenesis on spatial memory [28]. Moreover, through the use of these techniques, research has been performed in order to determine the role of exercise-induced adult neurogenesis on hippocampaldependent spatial memory. Still, these results are again mixed. For example, some focal irradiation studies report no requirement for exercise-induced hippocampal neurogenesis to mediate observed enhancement in the Morris water maze (MWM) [26, 29], while others show a loss of enhanced performance once new neurons were partially ablated [25]. Thus, an overall discrepancy in the literature exists, even within similar models, leaving the functional role of new neurons in spatial learning and memory unknown in both the sedentary and the exercising animal.

It is possible that the methods and techniques currently used cause side effects which may play a role in the discrepancy in the literature. For example, the irradiation method has limitations as it is known to induce inflammation in the brain that can be relatively long lasting [29]. Further, irradiation can also reduce or impair other undifferentiated or dividing cells in the areas of the brain and head exposed to radiation such as astrocytes, microglia and endothelial cells. It is possible that irradiation induces other defects in the brain, besides neurogenesis, which obliterate benefits that otherwise would be observed from exercise [25, 29].

Recently a transgenic mouse line was developed that allows selective, inducible reduction of adult neurogenesis. The transgene consists of a herpes simplex virus thymidine kinase (HSV-TK) under the control of a modified nestin promoter to restrict expression to neural progenitor cells [30]. The HSV-TK in the nestin positive cells (i.e., neural progenitors) phosphorylates exogenously administered ganciclovir resulting in a nucleotide analog that gets incorporated into newly synthesized DNA, and ultimately leads to termination of DNA elongation and apoptosis of the cell. In the present study, we evaluated performance of the nestin-TK transgene on a hybrid F1 genetic background consisting of C57BL/6J (B6) and 129S1/SvImJ (129) genotypes. The rationale for studying the transgene on a hybrid background is that the F1 offspring are isogenic (i.e., same sex individuals are genetically identical), but also heterozygous at all loci that differ between the two parental strains, and heterozygosity is typically associated with better overall health and behavioral performance.

Another novelty of our study was that instead of administering ganciclovir via surgically implanted minipumps which is the method usually employed with TK models, we administered valganciclovir via the chow. We have found that minipumps are invasive, and can cause skin lesions, or other discomfort, particularly when the animals are running on a running wheel. Valganciclovir gets converted into ganciclovir in the gut before reaching circulation and is preferable because administering via the chow is completely non-invasive $[30,31]$.

The novel F1 nestin-TK hybrid strain potentially could provide a powerful and minimally invasive model for reducing exercise-induced neurogenesis. However, no previous study has tested or evaluated the extent to which exercise-induced neurogenesis can be reduced using the nestin-TK model in either the B6 transgenic line or F1 hybrid. Therefore the purpose of this study is first to establish that reductions in adult neurogenesis will occur in both the B6 transgenic line and the novel hybrid F1 strain where mice are both housed under standard (sedentary) conditions or with running wheels. Second, we used the F1 nestinTK strain to directly test whether intact neurogenesis is required for spatial learning and memory on the MWM and rotarod test of motor performance. 


\section{MATERIALS AND METHODS}

\section{Subjects}

The Beckman Institute Animal Facility is AAALAC approved. All procedures were approved by the University of Illinois Institutional Animal Care and Use Committee and adhered to NIH guidelines (Protocol: 12140). In Experiment 1, a total of 80 female NestinTK mice with the C57BL/6J (B6) background were used. This included $n=34$ mice hemizygous for the nestin-TK transgene and $n=46$ non-transgenic control littermates [30]. In Experiment 2, a total of 223 (132 male, 91 female) mice from an F1 cross of B6 nestinTK with 129S1/SvImJ (129) were used. All mice were bred in house at the Beckman Institute and were genotyped to identify presence or absence of the transgene. The genotype of each mouse was verified prior to the start of the study by a tail snip followed by DNA extraction, PCR, and gel electrophoresis, using GAPDH as the control gene.

\section{Animal husbandry}

In Experiment 1, mice were born between 3/19/2012 and 4/02/2012, while mice from Experiment 2 were born between 9/27/2012 and 11/07/2012. All mice were weaned at postnatal day 21, group-housed four per cage by sex in standard polycarbonate shoebox cages with corncob bedding (Harlan Teklad, Madison, Wisconsin, USA) for approximately two to three months, then individually housed for six days just before the start of the study. Mice remained singly housed throughout the experiment. Therefore, mice from Experiment 1 were between 64 and 79 days old at the start of the experiment, while mice from Experiment 2 were between 63 and 104 days old at the start of the experiment. Individual mice from each litter were assigned to different groups, to account for litter effects or the possible impact of age. Throughout the study, mice were maintained on a reverse $12 \mathrm{~h}$ light/dark cycle (lights off at 10am CST, and on at 10pm) with food (Harlan Teklad 7012, until the start of the experiment) and water provided ad libitum.

\section{Valganciclovir administration}

For both Experiment 1 and Experiment 2, valganciclovir was administered orally, in the feed (chow). On day one of the experiment, their diet was switched from Harland Teklad 7012 to valganciclovir $(900 \mathrm{mg} / \mathrm{kg}$ of food) or control chow (both made by Custom Animal
Diets, Bangor, PA, USA). Chow was dyed either red (valganciclovir) or brown (control) in order for the animal care to visually differentiate between chow types and confirm that the animals were getting the correct chow type. Individual housing allowed tracking of the quantity of chow consumed per animal as measured by food disappearance. In Experiment 1, approximately $60 \mathrm{~g}$ of chow was added to each cage, weighed every 3 days, and replenished to an approximate mass of $60 \mathrm{~g}$ throughout the duration of the experiment. In Experiment 2, food was weighed every seven days (rather than every three). Therefore, a larger amount of chow (approximately $100 \mathrm{~g}$ ) was added to each cage to ensure mice had ample food throughout the week. Targeted consumption of valganciclovir per day was $200 \mathrm{mg} / \mathrm{kg}$ body mass [30].

\section{Exercise vs. sedentary treatments}

At the start of both experiments, concurrent with their diet switch, mice were assigned to either exercise or sedentary conditions. Table 1 describes the sample sizes for each experimental condition across Experiment. Additionally, for the first ten days in exercise or sedentary conditions, all mice received a daily intraperitoneal injection of $50 \mathrm{mg} / \mathrm{kg}$ BrdU (SigmaAldrich, St. Louis, MO, USA) in order to label dividing cells. Exercise conditions involved placement in a cage equipped with a 9-inch running wheel for thirty days, while sedentary conditions involved placement in standard polycarbonate boxes. In the exercise group, wheel rotations were monitored continuously in $1 \mathrm{~min}$ increments throughout the experiment via magnetic switches interfaced to a computer running VitalView software (Respironics, Bend, OR, USA). Sedentary mice were not kept in cages with locked wheels, as mice will climb on top of locked wheels and this would introduce additional activity in the sedentary group [32]. Housing in designated cages persisted throughout the behavioral testing except when temporarily moved into behavioral testing apparatuses. Wheel access continued throughout behavioral testing, until sacrifice of the mice in the runner groups, as removal of running wheels would introduce stress in the exercise group, and potentially confound behavioral testing results. An experimental timeline can be found in Fig. 1.

\section{Behavioral testing}

\section{$M W M$}

Mice from Experiment 2 were trained on MWM with 2 trials per day for 5 days. A trial lasted either $60 \mathrm{~s}$ 
Table 1

Sample sizes per group

\begin{tabular}{|c|c|c|c|c|c|c|c|c|}
\hline & NT Sed Control & NT Sed Val & NT Run Control & NT Run Val & T Sed Control & T Sed Val & T Run Control & T Run Val \\
\hline Exp. 1 & 11 & 12 & 11 & 12 & 8 & 9 & 9 & 8 \\
\hline Exp. 2 females & 12 & 12 & 12 & 12 & 10 & 11 & 11 & 11 \\
\hline Exp. 2 males & 15 & 15 & 15 & 16 & 17 & 18 & 18 & 18 \\
\hline
\end{tabular}

NT = non-transgenic; $\mathrm{T}=$ transgenic; Sed = sedentary; Run = runner; Val = valganciclovir.

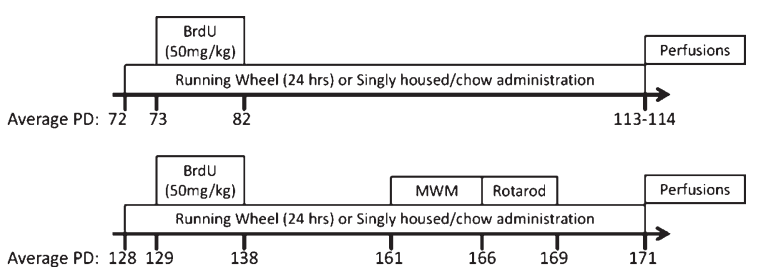

Fig. 1. Experimental timeline. Mice were bred in-house and weaned at 21 days of age, after which they were group housed by sex in cages of 4. Approximately 2 months later (A: Experiment 1 ) or 4 months later (B: Experiment 2), mice were individually housed, after which the transgenic and non-transgenic groups were each assigned to their conditions of exercise vs. sedentary and valganciclovir vs. control chow. These assignments coincided with the start of daily $50 \mathrm{mg} / \mathrm{kg}$ BrdU injections for 10 days. In Experiment 2, following 30 days on wheels, the animals were tested on the MWM for 5 days and the rotarod for 2 days.

or until the mouse reached the platform and remained on the platform for 5 seconds. If a mouse did not reach the platform in $60 \mathrm{~s}$, it was gently guided there by hand. Mice were placed back in their cages and allowed to rest for approximately 15 seconds between trials. Approximately 3 hours after training on Day 5 , the platform was removed and mice were tested with a probe trial lasting $60 \mathrm{~s}$. The maze consisted of a circular tub, $70 \mathrm{~cm}$ diameter and $30 \mathrm{~cm}$ deep. Extra-maze cues of varying geometric patterns, colors, and numbers were placed on the walls around the tub in order to provide spatial markers of location for the mice. A platform, made of a white plastic mesh measuring $8.5 \mathrm{~cm} \times 8.5 \mathrm{~cm}$, was placed in the middle of one maze quadrant and submerged $0.5 \mathrm{~cm}$ below the surface of the water. Crayola white tempera paint was added to the water to make the water sufficiently opaque to hide the platform from sight. White was chosen to provide contrast for video tracking from above (black mouse on white background). Water temperature was maintained at $24-26^{\circ} \mathrm{C}$. TopScan (CleverSystems, Reston, VA, USA) video tracking software was used to measure path length $(\mathrm{mm})$ to platform, and time (s) spent in the target quadrant of the maze during probe trial.

\section{Rotarod}

Animals were tested on a rotarod for three days (4 consecutive trials per day; AccuRotor Rota Rod Tall
Unit, $63 \mathrm{~cm}$ fall height, $8 \mathrm{~cm}$ diameter rotating dowel; Accuscan, Columbus, $\mathrm{OH}$ ). Animals were placed on the dowel starting at $0 \mathrm{rpm}$. The dowel was then accelerated at $60 \mathrm{rpm} / \mathrm{min}$. A photobeam at the base stopped the timer automatically when a mouse fell off the dowel onto bedding.

\section{Tissue preparation and immunohistochemistry}

Similar to previous work in our lab [25], animals were anesthetized with $100 \mathrm{mg} / \mathrm{kg}$ sodium pentobarbital via intraperitoneal injection to minimize suffering, and then perfused transcardially with $4 \%$ paraformaldehyde in phosphate buffer solution (PBS). Brains were postfixed overnight and transferred to $30 \%$ sucrose in PBS. Brains were sectioned using a cryostat into $40 \mu \mathrm{m}$ coronal sections and stored in tissue cryoprotectant at $-20^{\circ} \mathrm{C}$. Four separate 1-in-6 series of these sections (i.e., series of sections throughout the rostro-caudal extent of the brain with $240 \mu \mathrm{m}$ increments separating each section) were stained to visualize BrdU- diaminobenzidine (DAB) to detect newly divided cells in the dentate gyrus. Free-floating sections were washed in tris buffered saline (TBS) and then treated with $0.6 \%$ hydrogen peroxide. To denature DNA, sections were treated with $50 \%$ deionized formamide, $10 \% 20 \times$ saline-sodium citrate buffer, $2 \mathrm{~N}$ hydrochloric acid, and $0.1 \mathrm{M}$ boric acid. Sections were then treated with a solution of $0.1 \%$ Triton-X and $3 \%$ goat serum in TBS (TBS-X plus), and then incubated in monoclonal primary antibody against $\mathrm{BrdU}$ made in rat (OBT0030; Serotec, Raleigh, North Carolina, USA) at a dilution of 1:200 in TBS-X plus for $72 \mathrm{~h}$ at $48^{\circ} \mathrm{C}$. Sections were then washed in TBS, treated with TBS-X plus for $30 \mathrm{~min}$, and then incubated in secondary anti-rat antibody made in goat (BA-9400; Vector Laboratories, Burlingame CA, USA) at 1:250 in TBS-X plus for $100 \mathrm{~min}$ at room temperature. Sections were then treated using the Vectastain Elite ABC Kit (PK-6100; Vector Laboratories, Burlingame, CA, USA) and stained using a DAB kit (Sigma, St. Louis, MO, USA). Given the high $\mathrm{n}$ in each group in Experiment 2 , only 6 animals per group were stained and analyzed. 


\section{Image analysis}

As described previously [25], the entire bilateral granule layer, represented in the 1-in-6 series, was photographed by systematically advancing the field of view of the Zeiss brightfield light microscope and taking multiple photographs, via AxioCam interfaced to a computer, under $10 \times($ total $100 \times$ ) magnification. These photographs were then analyzed using ImageJ software (NIH, Bethesda, MD, USA) to generate unbiased estimates of total number of BrdU-labeled cells per cubic micrometer dentate gyrus sampled and area of the granular layer within the sections. Specifically, in each image, the granule layer was traced and BrdUpositive nuclei were counted within the traced region automatically by setting a fixed threshold to remove background. Total counts were multiplied by 6 to account for the 1-in- 6 series, and reduced by $15 \%$ to adjust for double counting at the plane of the section.

\section{Statistical analysis}

Data were analyzed using SAS version 9.2 and SPSS version 21. In all analyses, $p<0.05$ was considered statistically significant. The following variables were analyzed using a three-way analysis of variance (ANOVA), with genotype (transgenic vs. non-transgenic), exercise (runner vs. sedentary), and chow (valganciclovir vs. control) as the three factors, with all interactions entered in the model: chow intake (grams/day), valganciclovir dose ( $\mathrm{mg} / \mathrm{kg} / \mathrm{day})$ and duration (s) in the target quadrant of the MWM during the probe trial. In Experiment 1, the following dependent variables were transformed to improve assumptions of normality: total number of BrdUpositive cells (square root) in the dentate gyrus, and average distance run on wheels (log). In some cases, where stated, data were also analyzed by two-way ANOVA to determine genotype contributions to certain effects (e.g. within transgenic and non-transgenic groups for running levels, etc.).

\section{RESULTS}

\section{Wheel running}

In both experiments, running levels increased over the first 20 days and then leveled off. Female mice from Experiment 1, ran an average of $4.91 \mathrm{~km} /$ day $( \pm 0.27$ S.E.), while in Experiment 2, females ran an average of $5.66 \mathrm{~km} /$ day $( \pm 0.36 \mathrm{~S}$.E.), while males ran an average of $4.12 \mathrm{~km} /$ day $( \pm 0.27$ S.E.) (Exp. 2:
$\mathrm{F}_{1,107}=12.176, p<0.001$; Fig. 2A-C). In Experiment 1 , average running distance traveled over the entire period was significantly different between transgenic and non-transgenic animals. Transgenic animals ran approximately $80 \%$ as far as non-transgenic collapsed across chow type (Fig. $2 \mathrm{~B} ; \mathrm{F}_{1,36}=4.45$, $p=0.0419$ ). No significant differences in chow or the interaction between chow and genotype were detected. No differences in wheel running behavior between groups were evident in Experiment 2.

\section{Chow intake and valganciclovir dose}

In both experiments, the overall analysis indicated that mice housed with a running wheel ate more chow as compared to sedentary mice (Exp. 1 : $\mathrm{F}_{1,72}=85.28$, $p<0.0001$; Exp. 2: $\left.\mathrm{F}_{1,201}=51.77, p<0.0001\right)$. This was true both for the transgenic (Exp. 1: $\mathrm{F}_{1,30}=30.88$, $p<0.0001$; Exp. $\left.2: \mathrm{F}_{1,105}=28.99, p<0.0001\right)$ and nontransgenic mice (Exp. 1: $\mathrm{F}_{1,42}=59.38, p<0.0001$; Exp. 2: $\left.\mathrm{F}_{1,96}=22.99, p<0.0001\right)$, as more calories are needed to sustain aerobic exercise (Fig. 2D). In Experiment 1, in addition to the exercise effect, overall analysis of chow consumption showed a significant effect of chow type $\left(F_{1,72}=8.98, p=0.0037\right)$. This effect was largely influenced by the non-transgenic group, as there was a slight but significant increase in non-transgenic consumption of control chow as opposed to non-transgenic consumption of valganciclovir chow in runners $\left(\mathrm{F}_{1,42}=8.21, p=0.0065\right)$. Analysis of chow consumption within the transgenic group showed no effect of chow type. In Experiment 2, there was also a significant effect of chow type $\left(F_{1,201}=910.92, p=0.001\right)$. This effect was evident in both the transgenic $\left(\mathrm{F}_{1,105}=5.48, p=0.021\right)$ and nontransgenic animals $\left(\mathrm{F}_{1,96}=5.46, p=0.022\right)$ where a significant increase in consumption of control chow as opposed to valganciclovir chow was evident in runners. In both Experiment 1 and Experiment 2, the average valganciclovir dose acquired per day was also dependent upon exercise condition (Exp. 1: $\mathrm{F}_{1,37}=43.50$, $p<0.0001$; Exp. 2: $\mathrm{F}_{1,101}=66.07, p<0.0001$ ), but not genotype. Further, in Experiment 2 there was a main effect of $\operatorname{sex}\left(\mathrm{F}_{1,101}=77.83, p<0.0001\right)$, which resulted from females receiving an overall higher dose than males (Fig. 2E\&F).

\section{BrdU-DAB immunohistochemistry}

In Experiment 1, total number of BrdU+ cells in the granule layer depended on 1) whether animals were given access to a running wheel and 2) whether 


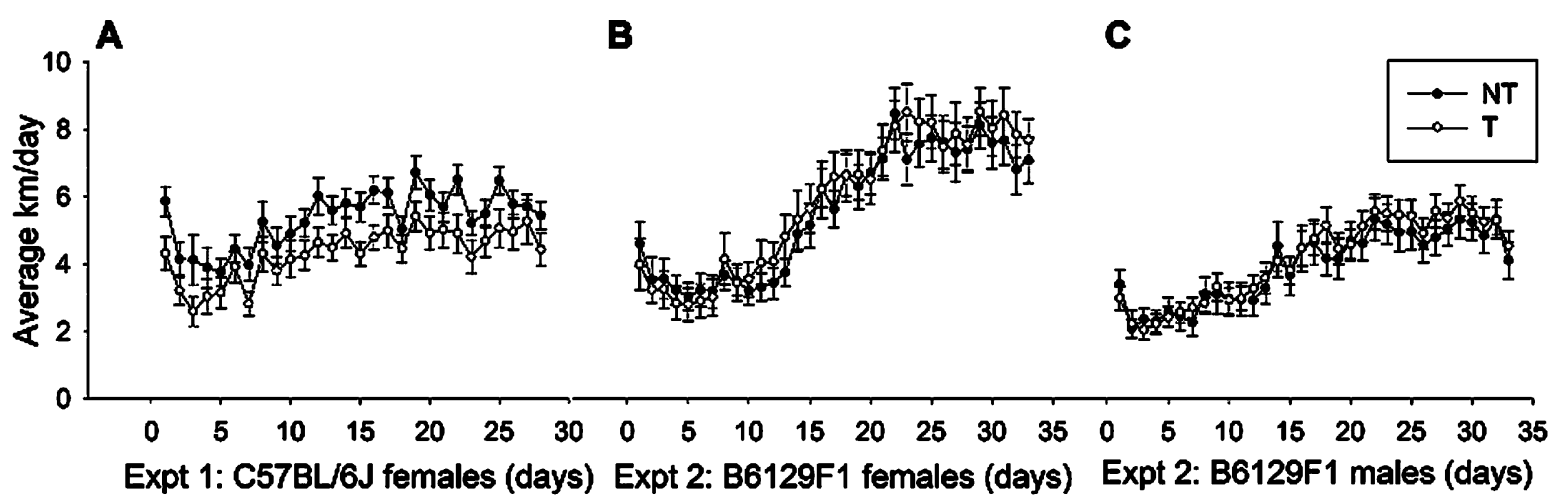

D

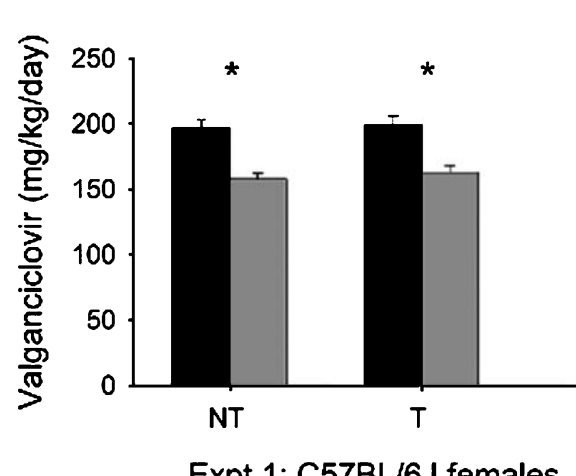

Expt 1: C57BL/6J females
$\mathbf{E}$

$\mathbf{F}$

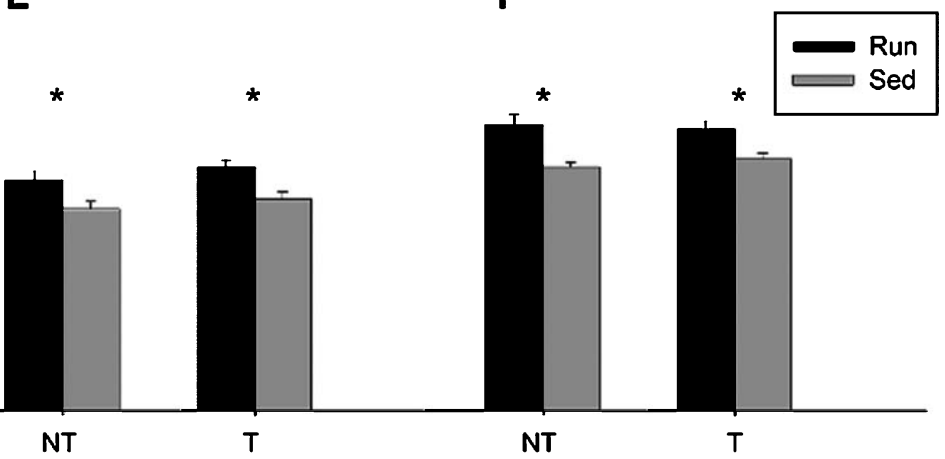

Expt 2: B6129F1 females

Expt 2: B6129F1 males

Fig. 2. Wheel running and valganciclovir dose. (A-C) Average distance run per day ( $\mathrm{km} /$ day) for each group varying by genotype (transgenic or non-transgenic). (D-F) Average valganciclovir dose per day ( $\mathrm{mg} / \mathrm{kg} / \mathrm{day})$ for valganciclovir-exposed groups. The average dose is estimated from the average grams of chow consumed per day, at $900 \mathrm{mg}$ valganciclovir/kg chow, and animal weight at sacrifice. Runners consumed significantly more chow, and hence received a higher dose as compared to sedentary animals. ${ }^{\wedge} p<0.01$. Values indicate means \pm SEM.

transgenic mice received the valganciclovir chow. In Experiment 1, an overall analysis of BrdU+ cells in the dentate showed a significant effect of genotype (Exp. 1: $\left.\mathrm{F}_{1,71}=18.91, p<0.0001\right)$, exercise $\left(\mathrm{F}_{1,71}=49.74\right.$, $p<0.0001)$, and chow $\left(\mathrm{F}_{1,71}=11.77, p=0.0010\right)$, as well as an interaction between genotype and exercise $\left(F_{1,71}=4.34, p=0.0407\right)$, and a trend for an interaction between exercise and chow $\left(\mathrm{F}_{1,71}=3.13\right.$, $p=0.0811$ ) (Fig. 3A). Access to a running wheel significantly increased the total number of BrdU+ cells in the dentate gyrus relative to sedentary animals in both the non-transgenic $\left(\mathrm{F}_{1,41}=49.46, p<0.0001\right)$ and the transgenic $\left(\mathrm{F}_{1,30}=12.61, p=0.0013\right)$ animals. As expected, no effect of chow was detected in the nontransgenic animals; however, a main effect of chow was present in the transgenic mice $\left(\mathrm{F}_{1,71}=14.81\right.$, $p=0.0006)$ was detected. As expected, transgenic mice (both runners and sedentary) that received valganciclovir exhibited a significant reduction in BrdU+ levels. In fact, post hoc tests revealed that exercise increased number of BrdU+ cells in the transgenic mice fed control chow but not valganciclovir chow (Fig. 3C-F). Thus administration of valganciclovir was able to halt exercise-induced adult neurogenesis, as the number of BrdU+ cells in transgenic runner mice receiving valganciclovir was similar to transgenic sedentary mice receiving control chow, and sedentary non-transgenic mice receiving either chow type.

Experiment 2 expanded on the results of Experiment 1 by examining levels of adult neurogenesis in the F1 hybrid nestin-TK mice that are isogenic but also heterozygous at all loci that differ between the parental strains. Consistent with previous strains, total number of BrdU+ cells in the granule layer depended on 1) whether animals were given access to a running wheel and 2) whether transgenic mice received the valganciclovir chow. An overall analysis of BrdU+ cells in the dentate of transgenic animals showed both an interaction between chow and exercise $\left(\mathrm{F}_{1,39}=46.27\right.$, $p=0.017)$ and an interaction between sex and exercise $\left(\mathrm{F}_{1,39}=7.23, p=0.010\right)$. In addition, a significant main effect of exercise $\left(F_{1,39}=34.32, p<0.0001\right)$, wherein 


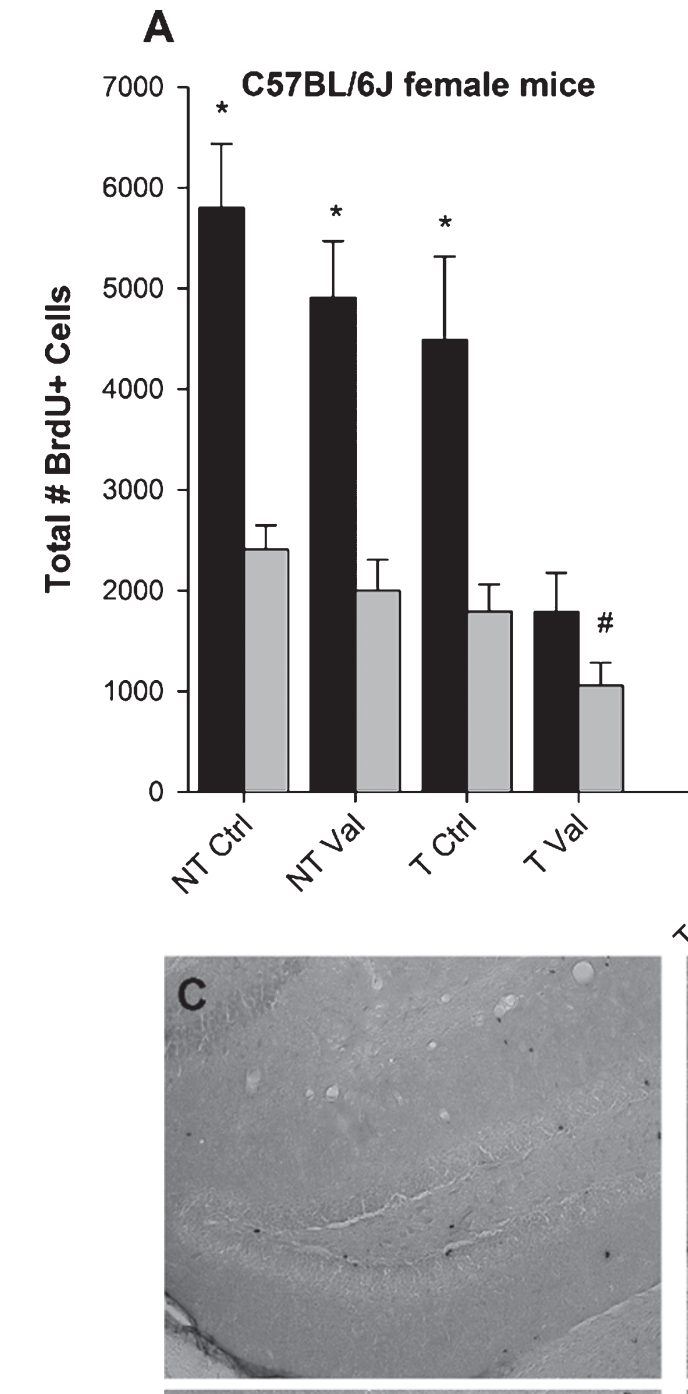

B

B6129F1 male and female mice
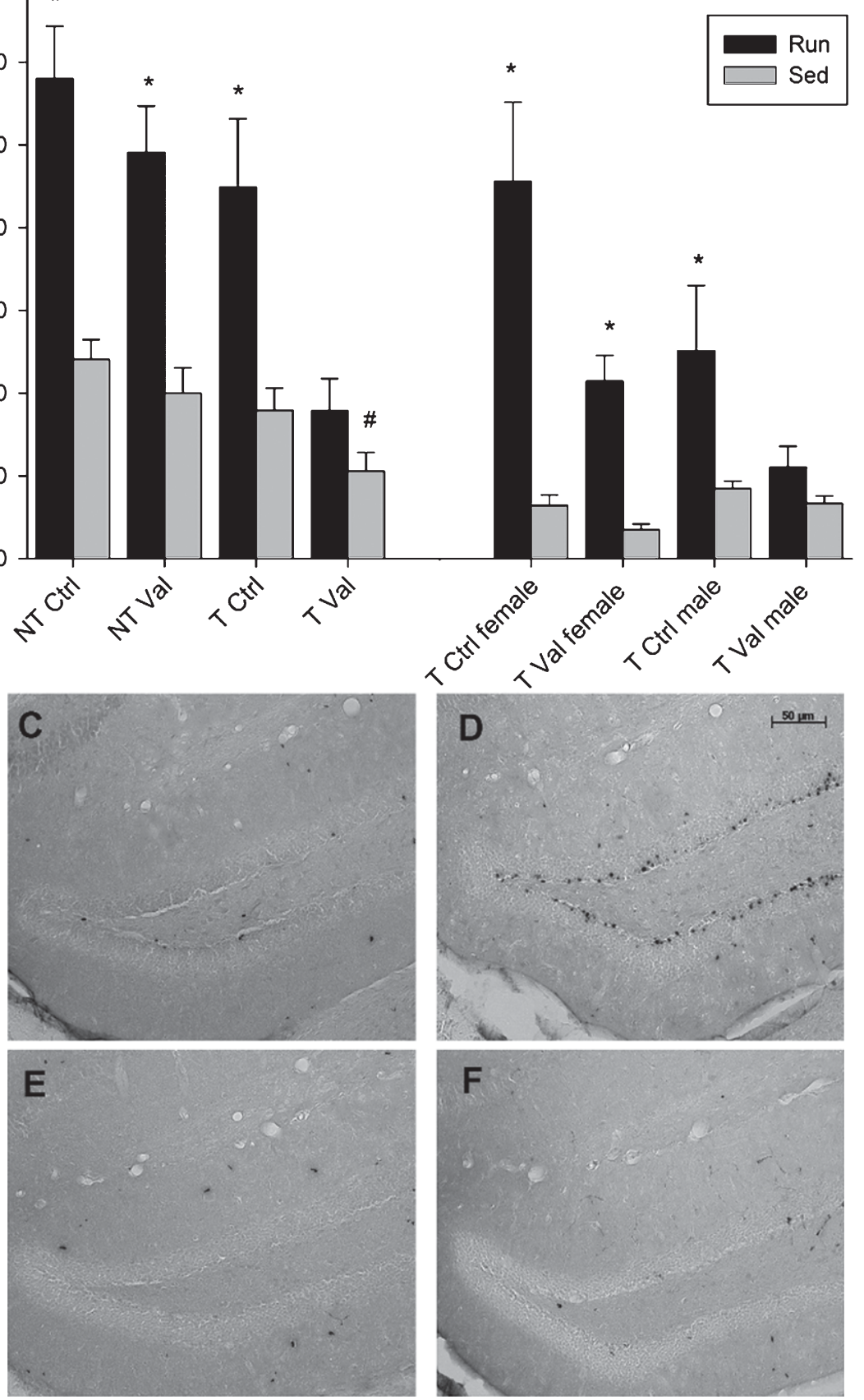

Fig. 3. Adult hippocampal neurogenesis. Total number of BrdU-positive cells in the granule layer of the dentate gyrus detected by BrdU-DAB for animals in Experiment 1 on the B6 background (A) and Experiment 2 on the F1 background (B). (C-F). Images represent BrdU immunostaining in hippocampal dentate gyrus from sample animals from each group from the F1 model: transgenic sedentary fed control chow (C), transgenic runner fed control chow (D), transgenic runner fed valganciclovir chow (E) and transgenic sedentary fed valganciclovir chow (F). ${ }^{*} p<0.05$, ${ }^{\#} p<0.05$ compared to sedentary NT-Ctrl. Values indicate means \pm SEM. 
access to a running wheel produced higher levels of BrdU+ cells was evident. Results also indicated a main effect of chow $\left(\mathrm{F}_{1,39}=10.33, p=0.003\right)$, in which transgenic mice that received valganciclovir had significantly fewer BrdU+ cells than did those that received control chow. Finally, a trend for a main effect of sex $\left(\mathrm{F}_{1,39}=3.71, p=0.061\right.$; Fig. $\left.3 \mathrm{~B}\right)$ was also observed. Post hoc test revealed that transgenic runners fed control chow had significantly higher levels of BrdU+ cells when compared with all other groups (TRunVal: $p=0.0001$, TSedControl: $p<0.0001$, TSedVal: $p<0.0001)$. Together, these data indicate that $900 \mathrm{mg}$ of valganciclovir per $\mathrm{kg}$ of chow is sufficient to reduce new exercise-induced neural cells to baseline sedentary levels.

\section{BrdU+cells and valganciclovir dose correlations}

In Experiment 1, correlation analyses were performed to determine the relationship between number of BrdU+ cells and dose of valganciclovir consumed, but no statistical significance was reached. In fact, in runners, the relationship was slightly positive and in the opposite direction as expected. Although not significant, it is possible that a true positive relationship exists within this range of chow eaten because more chow eaten was correlated with greater wheel running, which is causally related to increased numbers of BrdU+ cells. More importantly, the result of no significant correlation between dose of valganciclovir consumed and number of new neurons within the transgenic mice, or a slightly positive relationship, suggests that the small differences in valganciclovir consumed between runners and sedentary animals, due to amount of chow eaten, was not significant enough to impact the total number of new cells. In other words, the differences in number of new cells between the runner and sedentary mice we detected in this study are unlikely to be due to subtle differences in the dose of valganciclovir received. Results from Experiment 2 indicate a similar lack of correlation between the number of BrdU+ cells and dose of valganciclovir consumed as well as a slightly positive correlation in the runners.

\section{$M W M$}

In Experiment 2, an overall analysis of distance traveled to reach the platform across days revealed a main effect of day $\left(\mathrm{F}_{1,178}=910.27, p<0.0001\right)$, a main effect of exercise $\left(\mathrm{F}_{1,178}=11.45, p=0.001\right)$ and a sex by genotype interaction $\left(\mathrm{F}_{1,178}=4.60\right.$, $p=0.033$ ). Results demonstrate that runners traveled a significantly longer distance to reach the platform on Day $1\left(\mathrm{~F}_{1,192}=21.42, p<0.0001\right)$ and Day $2\left(\mathrm{~F}_{1,194}=20.89, p<0.0001\right)$ of MWM acquisition, suggesting the animals may not have learned the task as quickly as sedentary animals (Fig. 4A). No significant difference in swim speed was evident between exercise groups (Fig. 4B). Further, there was no difference in distance traveled between nontransgenic males and females; however, a significant difference was evident between transgenic males and females $\left(F_{1,90}=5.25, p=0.024\right)$ wherein females traveled an overall greater distance to reach the platform than did males $(5375.76 \mathrm{~mm} \pm 243.23 \mathrm{~mm}$ versus $4660.36 \mathrm{~mm} \pm 195.73 \mathrm{~mm}$ ). An overall analysis of the number of bouts in the target quadrant during the probe trial revealed a main effect of exercise $\left(\mathrm{F}_{1,180}=26.874\right.$, $p=0.009)$, with runners outperforming sedentary animals, specifically the females $\left(\mathrm{F}_{1,74}=4.55, p=0.036\right.$; Fig. 4C). Interestingly, no difference on time spent in each quadrant was evident (Fig. 4D).

\section{Rotarod}

In Experiment 2, an overall analysis of time spent on the rotarod on each of the twelve trials revealed a significant main effect of $\operatorname{sex}\left(\mathrm{F}_{1,202}=8.72, p=0.004\right)$ as well as a sex by exercise interaction $\left(\mathrm{F}_{1,202}=4.02\right.$, $p=0.046$ ). Specifically, in females there was a main effect of exercise $\left(\mathrm{F}_{1,81}=4.85, p=0.030\right.$; Fig. 5A) wherein runners were able to stay on the rotarod for a longer time than sedentary animals. This effect was not evident in males (Fig. 5B).

\section{DISCUSSION}

The present study assessed the functionality of the nestin-TK mouse model in a novel hybrid C57BL/6 J x $129 \mathrm{~S} 1 / \mathrm{SvImJ}$ background to test the role of newly generated neurons in spatial learning and memory and motor performance on the rotarod. The results demonstrate that intact adult hippocampal neurogenesis is not required for performance on either task. Specifically, valganciclovir chow administration in the F1 nestin-TK animals significantly reduced neurogenesis by approximately one half (Fig. 3B). However, even though the mice displayed reduced neurogenesis, their performance on the tasks was similar to the animals with intact neurogenesis (Figs. 4\&5). These findings are the first to demonstrate that new neurons are not required for MWM or rotarod performance in the F1 hybrid mouse strain nor are they required for any of the subtle effects of exercise that were detected in the water 

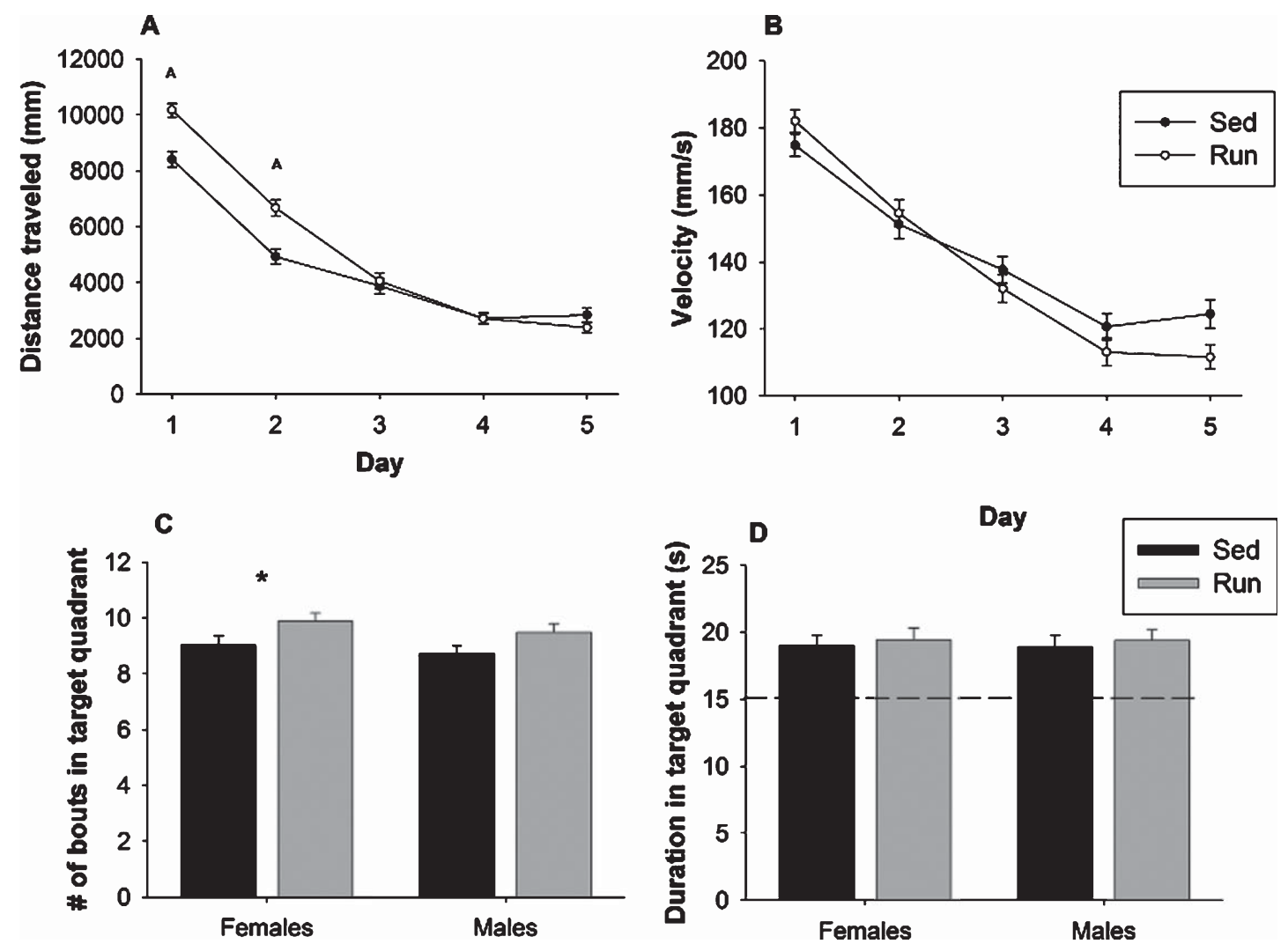

Fig. 4. MWM performance. (A) Acquisition of the MWM task in path length ( $\mathrm{mm}$ ) in sedentary and runners, as the average path length of two trials per day. There exists a counterintuitive effect of exercise in acquisition, as sedentary mice outperformed runners in path length to platform on Day 1 and Day 2. (B) Average velocity ( $\mathrm{mm} / \mathrm{s}$ ) across two trials per day of both runners and sedentary animals. (C-D) Probe trial data for the MWM, represented as number of bouts (C) and duration (D) in the target quadrant indicates that although runners crossed into the target quadrant significantly more than did sedentary animals, all animals appeared to learn the task as each group spent over 15 seconds in the target quadrant. ${ }^{\wedge} p<0.01,{ }^{*} p<0.05$. Values indicate means \pm SEM.
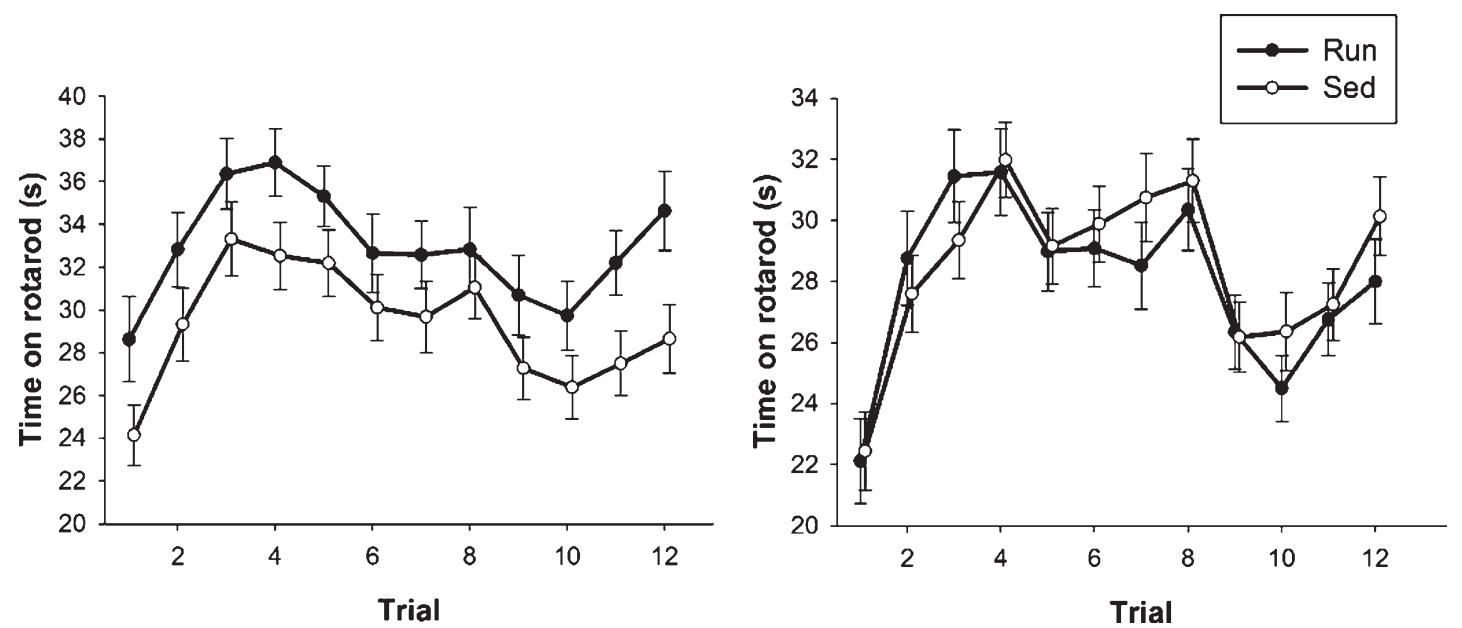

Fig. 5. Rotarod performance. Average duration (s) spent on the rotarod for both females (A) and males (B). Performance is represented as data from each individual trial across the 2 days of rotarod testing. Values indicate means \pm SEM. 
maze or rotarod in this strain. Results for rotarod were expected because performance on the rotarod is not dependent on the hippocampus [33]. However, results for the MWM were not necessarily expected as many studies have suggested that new neurons play a critical role in spatial learning and memory, specifically in the MWM [25, 34-37].

Although runners consumed slightly more food and hence received a slightly higher dose of valganciclovir as compared to sedentary animals (Fig. 2D-F), this small difference in dose is unlikely to have contributed significantly to levels of neurogenesis in the animals for two reasons. First, the amount ingested was not significantly correlated with number of BrdU+ cells in the transgenic animals, indicating either that there was a threshold dose that once exceeded results in the same maximal reduction of neurogenesis, or that the differences were too small to be detectable. Second, the transgenic runners displayed higher levels of neurogenesis than transgenic sedentary animals (Fig. 3) even though the runners received a higher dose of valganciclovir. Therefore, differences in neurogenesis between runner and sedentary mice that received valganciclovir cannot be accounted for by the dose.

Unfortunately, we did not observe a clear enhancement in performance on the MWM from exercise in the control-fed hybrid F1 nestin-TK transgenic mice. Previously, we published a study using irradiation to reduce neurogenesis in the $\mathrm{C} 57 \mathrm{BL} / 6 \mathrm{~J}$ genotype, and found that the enhancement in learning and memory on the MWM was abolished in irradiated animals with reduced neurogenesis [25]. Hence, we were hoping to confirm the finding using the more specific nestinTK transgenic model since irradiation can induce side effects such as inflammation that itself can affect function and account for the behavioral deficits [38, 39]. However, overall, in the current study, the runners took longer to acquire the task (Fig. 4A), and spent a similar time in the target quadrant during the probe test (Fig. 4D). They displayed significantly more crosses through the target quadrant during the probe test (Fig. 4C), which might be taken as evidence of more accurate spatial memory. However, because of the apparent impaired performance during acquisition and no difference in duration spent in the target quadrant during the probe test, the benefits, if any, on MWM performance from wheel running in the F1 hybrid were small at best.

Many studies across species have shown benefits of exercise on MWM performance or other measures of spatial learning and memory [20, 21, 40-48]. Therefore, the explanation of why the effect was not replicated for the F1 hybrid in our study is not clear. To the best of our knowledge, the standard water maze has never been performed in the B $6 \times 129 \mathrm{~S} 1$ hybrid background. Each strain is known to respond differently to the MWM [49]. Recently we found that the F1 hybrid does in fact display enhanced performance from exercise if tested in the plus version of the water maze, specifically adapted for use in multi-strain comparisons [46]. Hence, the standard water maze parameters used in our study may simply not have been optimal to detect exercise effects in the F1 hybrid. Since the F1 hybrid displayed increased neurogenesis from exercise, it represents another illustration that new neurons are not sufficient to improve learning and memory [50]. On the other hand, results suggest that the F1 hybrid may not be ideal to test the functional significance of new neurons in improved performance on standard versions of the MWM that work well in other strains [21, 40, 44, 45, 51, 52].

We concluded that intact neurogenesis is not required for baseline performance or subtle differences in performance induced from voluntary wheel running exercise in our model. However, it is important to note that our method of reducing neurogenesis did not result in a complete ablation of neurogenesis. Therefore, it is possible that had neurogenesis been completely ablated we would have seen an impact on the behavioral outcomes. Nevertheless, the study shows that intact neurogenesis is not required for learning and that neurogenesis can at least be reduced by $50 \%$ without affecting learning on the water maze.

The hybrid B6129SF1/J background mouse strain was selected due to previously documented high levels of running (approximately $10 \mathrm{~km} /$ day) and high levels of adult neurogenesis in runners compared to sedentary animals [53]. However, the animals in the current study failed to reach similar high levels of wheel running. One possibility for the slightly reduced running is the weighing of the food, which may have disturbed the animals more than usual. It is also possible that the transgene unintentionally disrupted circuitry related to motivation for running. It is also possible that the difference represents environmental variation related to the mouse handler, season, humidity or other uncontrolled variables between studies. Regardless of the reason, the slightly lower levels of running in the F1 hybrid than expected in our study may have contributed to the lack of a clear improvement on the MWM from wheel running.

Previous methods for administering ganciclovir to nestin-TK mouse and rat strains have typically used osmotic mini-pumps which must be surgically 
implanted under the skin, and can cause skin lesions (personal observation). The method of administering valganciclovir via the chow is completely noninvasive, and as we demonstrate in this paper works well for reducing neurogenesis in the nestin-TK models. The $50 \%$ reduction in neurogenesis in the transgenic mice is similar to what we previously reported using this method [54]. Valganciclovir is the ester prodrug form of ganciclovir that rapidly converts to ganciclovir in the gut via hepatic esterases. The reduction in BrdU+ cells reported in the current study is not as robust as the $90 \%$ ablation reported by mini-pump delivery of ganciclovir in the Kernie group [30] or the $98 \%$ reduction exhibited by the Flint group [28]. However, the advantage is that chow-administered valganciclovir does not require anesthesia or mini-pump implantation surgery; factors that may further reduce neurogenesis and cause other side effects that could impair behavioral performance. Hence, the results establish the validity of using valganciclovir via the chow for reducing neurogenesis in the F1 and C57BL/6J nestin-TK models consistent with other studies that have used this method in the B6 background [54]. Further we conclude after having experience with both methods that the chowadministered valganciclovir is better because of the non-invasiveness for evaluating the specific contribution of new neurons in behavior.

Our finding that intact neurogenesis is not required for normal baseline performance on the MWM is consistent with a growing number of studies reporting that neurogenesis reduction has no influence on behavior $[28,29,54]$. The result is consistent with our previous report using irradiation to reduce neurogenesis, because the neurogenesis reduction in that study did not impair baseline performance on the MWM; it only abolished the enhancement in performance from exercise [25]. As mentioned previously, the enhancement in performance could not be addressed in our study because no clear pro-cognitive effect of exercise was observed in the F1 genotype employed here (Fig. 4).

Although a growing number of studies using highly specific methods for reducing neurogenesis such as the nestin- or GFAP-TK mice are finding that new neurons are not required for hippocampus-dependent spatial learning and memory [28, 29, 54, 55], some studies are finding positive results, usually when baseline performance is reduced from injury. For example, work from the Kernie group examining the causal role of newly generated neurons in the recovery of poststroke cognitive function induced an experimental stroke in B6 nestin-TK transgenic mice by occluding the distal middle cerebral artery. Transgenic stroke mice administered ganciclovir through osmotic mini-pumps exhibited impaired performance on the Barnes Maze test when compared to saline control or wild-type stroke mice given ganciclovir [39], implicating a causal role between newly generated neurons and cognitive function. Further, nestin-TK animals with neurogenesis ablated, specifically at the time of a traumatic brain injury, are unable to learn spatial memory tasks, including the MWM [30]. Finally, through the use of the nestin-TK, Deng and colleagues [27] were able to specifically reduce the number of adult-born cells at particular maturation stages. In doing so, they demonstrated that, in particular, a reduction in the number of immature neurons led to impaired performance on the MWM.

Although our study and a growing number of others suggest that new neurons are not required for learning or memory on the MWM in otherwise intact animals $[26,28,29]$, we are not ready to give up the hypothesis that the new neurons do in fact contribute to learning and memory on this task or any other task that involves the hippocampus. Our revised hypothesis is that while new neurons may not be necessary they may be preferentially involved, similar to the way the hippocampus is not necessary for contextual fear conditioning, but is critically involved [56-58]. In other words, in animals that have neurogenesis reduced, other mechanisms of plasticity such as additional synapse formation may compensate for the loss. Recently it was discovered that suppression of adult neurogenesis through a nestin-TK transgenic model resulted in deficits in LTP, specifically in response to medial perforant path stimulation [59]. Interestingly, LTP recovery was accompanied by an increase in the survival rate of newborn cells that were proliferating just before the ablation and a reduction in inhibitory input to the granule cells of the dentate gyrus [59]. Therefore, it is possible that altered hippocampal connectivity may compensate for the decreased presence of newly generated neurons and allow for the network to function properly.

One way to get around the problem of compensatory effects from reducing neurogenesis is to use an approach that inactivates rather than completely eliminates new neurons. Such a strategy could be implemented using designer receptors exclusively activated by designer drugs (DREADDS) [60] or optogenetic manipulation of new neurons [61] that would allow temporary inactivation of newly generated dentate granule cells during key phases of the learning and memory process. We conclude that transgenic 
methods for manipulating the number and function of new neurons hold promise for uncovering the functional significance of adult hippocampal neurogenesis in behavior.

\section{ACKNOWLEDGMENTS}

This work was supported by NIH RO1 grants MH083807 (J.S.R), and DA027487 (J.S.R.). The authors would like to extend their sincere gratitude to the Beckman Institute Animal Facility staff for excellent animal care. They would also like to thank Tushar Bhattacharya, Martina Mustroph and Robert Holland for their help with the collection of behavioral data and also Heinrich Pinardo and Aya Kobeissi for their help with the imaging of hippocampal tissue.

\section{REFERENCES}

[1] Altman J. Autoradiographic and histological studies of postnatal neurogenesis. IV. Cell proliferation and migration in the anterior forebrain, with special reference to persisting neurogenesis in the olfactory bulb. J Comp Neurol. 1969;137:433-57.

[2] Altman J, Das GD. Autoradiographic and histological studies of postnatal neurogenesis. I. A longitudinal investigation of the kinetics, migration and transformation of cells incorporating tritiated thymidine in neonate rats, with special reference to postnatal neurogenesis in some brain regions. J Comp Neurol. 1966;126:337-89.

[3] Eriksson PS, Perfilieva E, Bjork-Eriksson T, Alborn AM, Nordborg C, et al. Neurogenesis in the adult human hippocampus. Nat Med. 1998;4:1313-7.

[4] Gould E, Tanapat P, McEwen BS, Flugge G, Fuchs E. Proliferation of granule cell precursors in the dentate gyrus of adult monkeys is diminished by stress. Proc Natl Acad Sci U S A. 1998;95:3168-71.

[5] Kaplan MS, Hinds JW. Neurogenesis in the adult rat: Electron microscopic analysis of light radioautographs. Science. 1977;197:1092-4.

[6] Ge S, Pradhan DA, Ming GL, Song H. GABA sets the tempo for activity-dependent adult neurogenesis. Trends Neurosci. 2007;30:1-8

[7] Kee N, Teixeira CM, Wang AH, Frankland PW. Preferential incorporation of adult-generated granule cells into spatial memory networks in the dentate gyrus. Nat Neurosci. 2007; 10:355-62

[8] Toni N, Laplagne DA, Zhao C, Lombardi G, Ribak CE, et al. Neurons born in the adult dentate gyrus form functional synapses with target cells. Nat Neurosci. 2008;11:901-7.

[9] van Praag H, Schinder AF, Christie BR, Toni N, Palmer TD, et al. Functional neurogenesis in the adult hippocampus. Nature. 2002;415:1030-4.

[10] Schmidt-Hieber C, Jonas P, Bischofberger J. Enhanced synaptic plasticity in newly generated granule cells of the adult hippocampus. Nature. 2004;429:184-7.

[11] Tashiro A, Makino H, Gage FH. Experience-specific functional modification of the dentate gyrus through adult neurogenesis: A critical period during an immature stage. J Neurosci. 2007;27:3252-9.
[12] Neeper SA, Gomez-Pinilla F, Choi J, Cotman C. Exercise and brain neurotrophins. Nature. 1995;373:109.

[13] Clark PJ, Bhattacharya TK, Miller DS, Kohman RA, DeYoung EK, et al. New neurons generated from running are broadly recruited into neuronal activation associated with three different hippocampus-involved tasks. Hippocampus. 2012;22:1860-7.

[14] Dabe EC, Majdak P, Bhattacharya TK, Miller DS, Rhodes JS. Chronic D-amphetamine administered from childhood to adulthood dose-dependently increases the survival of new neurons in the hippocampus of male $\mathrm{C} 57 \mathrm{BL} / 6 \mathrm{~J}$ mice. Neuroscience. 2013;231:125-35.

[15] Duman RS. Depression: A case of neuronal life and death? Biol Psychiatry. 2004;56:140-5.

[16] Hamilton GF, Boschen KE, Goodlett CR, Greenough WT, Klintsova AY. Housing in environmental complexity following wheel running augments survival of newly generated hippocampal neurons in a rat model of binge alcohol exposure during the third trimester equivalent. Alcohol Clin Exp Res. 2012;36:1196-204.

[17] Ma DK, Kim WR, Ming GL, Song H. Activity-dependent extrinsic regulation of adult olfactory bulb and hippocampal neurogenesis. Ann N Y Acad Sci. 2009;1170:664-73.

[18] Pham K, Nacher J, Hof PR, McEwen BS. Repeated restraint stress suppresses neurogenesis and induces biphasic PSANCAM expression in the adult rat dentate gyrus. Eur $\mathrm{J}$ Neurosci. 2003;17:879-86.

[19] van Praag H, Kempermann G, Gage FH. Running increases cell proliferation and neurogenesis in the adult mouse dentate gyrus. Nat Neurosci. 1999;2:266-70.

[20] Van der Borght K, Havekes R, Bos T, Eggen BJ, Van der Zee EA. Exercise improves memory acquisition and retrieval in the Y-maze task: Relationship with hippocampal neurogenesis. Behav Neurosci. 2007;121:324-34.

[21] van Praag H, Christie BR, Sejnowski TJ, Gage FH. Running enhances neurogenesis, learning, and long-term potentiation in mice. Proc Natl Acad Sci U S A. 1999;96:13427-31

[22] Kramer AF, Erickson KI. Capitalizing on cortical plasticity: Influence of physical activity on cognition and brain function. Trends Cogn Sci. 2007;11:342-8.

[23] Shors TJ, Miesegaes G, Beylin A, Zhao M, Rydel T, et al. Neurogenesis in the adult is involved in the formation of trace memories. Nature. 2001;410:372-6.

[24] Snyder JS, Hong NS, McDonald RJ, Wojtowicz JM. A role for adult neurogenesis in spatial long-term memory. Neuroscience. 2005; 130:843-52.

[25] Clark PJ, Brzezinska WJ, Thomas MW, Ryzhenko NA, Toshkov SA, et al. Intact neurogenesis is required for benefits of exercise on spatial memory but not motor performance or contextual fear conditioning in C57BL/6J mice. Neuroscience. 2008; $155: 1048-58$

[26] Saxe MD, Battaglia F, Wang JW, Malleret G, David DJ, et al. Ablation of hippocampal neurogenesis impairs contextual fear conditioning and synaptic plasticity in the dentate gyrus. Proc Natl Acad Sci U S A. 2006;103:17501-6.

[27] Deng W, Saxe MD, Gallina IS, Gage FH. Adult-born hippocampal dentate granule cells undergoing maturation modulate learning and memory in the brain. $\mathrm{J}$ Neurosci. 2009;29:13532-42.

[28] Groves JO, Leslie I, Huang GJ, McHugh SB, Taylor A, et al. Ablating adult neurogenesis in the rat has no effect on spatial processing: Evidence from a novel pharmacogenetic model. PLoS Genet. 2013;9:e1003718.

[29] Meshi D, Drew MR, Saxe M, Ansorge MS, David D, et al. Hippocampal neurogenesis is not required for behavioral 
effects of environmental enrichment. Nat Neurosci. 2006;9: 729-31.

[30] Blaiss CA, Yu TS, Zhang G, Chen J, Dimchev G, et al. Temporally specified genetic ablation of neurogenesis impairs cognitive recovery after traumatic brain injury. J Neurosci. 2011;31:4906-16.

[31] Chen J, Li Y, Yu TS, McKay RM, Burns DK, et al. A restricted cell population propagates glioblastoma growth after chemotherapy. Nature. 2012;488:522-6.

[32] Koteja P, Garland T, Jr., Sax JK, Swallow JG, Carter PA. Behaviour of house mice artificially selected for high levels of voluntary wheel running. Anim Behav. 1999;58: 1307-18.

[33] Goddyn H, Leo S, Meert T, D'Hooge R. Differences in behavioural test battery performance between mice with hippocampal and cerebellar lesions. Behav Brain Res. 2006; 173:138-47.

[34] Dupret D, Fabre A, Dobrossy MD, Panatier A, Rodriguez JJ, et al. Spatial learning depends on both the addition and removal of new hippocampal neurons. PLoS Biol. 2007;5:e214.

[35] Garthe A, Behr J, Kempermann G. Adult-generated hippocampal neurons allow the flexible use of spatially precise learning strategies. PLoS One. 2009;4:e5464.

[36] Gil-Mohapel J, Brocardo PS, Choquette W, Gothard R, Simpson JM, et al. Hippocampal neurogenesis levels predict WATERMAZE search strategies in the aging brain. PLoS One. 2013;8:e75125.

[37] Jessberger S, Clark RE, Broadbent NJ, Clemenson GD, Jr., Consiglio A, et al. Dentate gyrus-specific knockdown of adult neurogenesis impairs spatial and object recognition memory in adult rats. Learn Mem. 2009;16:147-54.

[38] Monje ML, Toda H, Palmer TD. Inflammatory blockade restores adult hippocampal neurogenesis. Science. 2003;302:1760-5.

[39] Sun C, Sun H, Wu S, Lee CC, Akamatsu Y, et al. Conditional ablation of neuroprogenitor cells in adult mice impedes recovery of poststroke cognitive function and reduces synaptic connectivity in the perforant pathway. J Neurosci. 2013;33:17314-25.

[40] Patil SS, Sunyer B, Hoger H, Lubec G. Evaluation of spatial memory of C57BL/6J and CD1 mice in the Barnes maze, the Multiple T-maze and in the Morris water maze. Behav Brain Res. 2009;198:58-68.

[41] Churchill JD, Galvez R, Colcombe S, Swain RA, Kramer $\mathrm{AF}$, et al. Exercise, experience and the aging brain. Neurobiol Aging. 2002;23:941-55

[42] Colcombe SJ, Kramer AF, Erickson KI, Scalf P, McAuley E et al. Cardiovascular fitness, cortical plasticity, and aging. Proc Natl Acad Sci U S A. 2004;101:3316-21.

[43] Nichol K, Deeny SP, Seif J, Camaclang K, Cotman CW. Exercise improves cognition and hippocampal plasticity in APOE epsilon4 mice. Alzheimers Dement. 2009;5:287-94.

[44] Fordyce DE, Wehner JM. Physical activity enhances spatial learning performance with an associated alteration in hippocampal protein kinase $\mathrm{C}$ activity in $\mathrm{C} 57 \mathrm{BL} / 6$ and $\mathrm{DBA} / 2$ mice. Brain Res. 1993;619:111-9.

[45] van Praag H, Shubert T, Zhao C, Gage FH. Exercise enhances learning and hippocampal neurogenesis in aged mice. J Neurosci. 2005;25:8680-5.
[46] Merritt JR, Rhodes JS. Mouse genetic differences in voluntary wheel running, adult hippocampal neurogenesis and learning on the multi-strain-adapted plus water maze. Behav Brain Res. 2015;280:62-71.

[47] Anderson BJ, Rapp DN, Baek DH, McCloskey DP, CoburnLitvak PS, et al. Exercise influences spatial learning in the radial arm maze. Physiol Behav. 2000;70:425-9.

[48] Alaei H, Moloudi R, Sarkaki AR, Azizi-Malekabadi H, Hanninen O Daily running promotes spatial learning and memory in rats. Pathophysiology. 2007;14:105-8.

[49] Wahlsten D, Cooper SF, Crabbe JC. Different rankings of inbred mouse strains on the Morris maze and a refined 4-arm water escape task. Behav Brain Res. 2005;165:36-51.

[50] Rhodes JS, van Praag H, Jeffrey S, Girard I, Mitchell GS, et al. Exercise increases hippocampal neurogenesis to high levels but does not improve spatial learning in mice bred for increased voluntary wheel running. Behav Neurosci. 2003;117:1006-16

[51] Mustroph ML, Chen S, Desai SC, Cay EB, DeYoung EK, et al. Aerobic exercise is the critical variable in an enriched environment that increases hippocampal neurogenesis and water maze learning in male C57BL/6J mice. Neuroscience. 2012;219:62-71.

[52] D'Hooge R, De Deyn PP. Applications of the Morris water maze in the study of learning and memory. Brain Res Brain Res Rev. 2001;36:60-90.

[53] Clark PJ, Kohman RA, Miller DS, Bhattacharya TK, Brzezinska WJ, et al. Genetic influences on exercise-induced adult hippocampal neurogenesis across 12 divergent mouse strains. Genes Brain Behav. 2011;10:345-53.

[54] Mustroph ML, Merritt JR, Holloway AL, Pinardo H, Miller DS, et al. Increased adult hippocampal neurogenesis is not necessary for wheel running to abolish conditioned place preference for cocaine in mice. Eur J Neurosci. 2015;41: 216-26.

[55] Hernandez-Rabaza V, Llorens-Martin M, VelazquezSanchez C, Ferragud A, Arcusa A, et al. Inhibition of adult hippocampal neurogenesis disrupts contextual learning but spares spatial working memory, long-term conditional rule retention and spatial reversal. Neuroscience. 2009;159:59-68.

[56] Gewirtz JC, McNish KA, Davis M. Is the hippocampus necessary for contextual fear conditioning? Behav Brain Res. 2000;110:83-95.

[57] Sanders MJ, Wiltgen BJ, Fanselow MS. The place of the hippocampus in fear conditioning. Eur $\mathrm{J}$ Pharmacol. 2003:463:217-23.

[58] Wiltgen BJ, Sanders MJ, Anagnostaras SG, Sage JR, Fanselow MS. Context fear learning in the absence of the hippocampus. J Neurosci. 2006;26:5484-91.

[59] Singer BH, Gamelli AE, Fuller CL, Temme SJ, Parent JM, et al. Compensatory network changes in the dentate gyrus restore long-term potentiation following ablation of neurogenesis in young-adult mice. Proc Natl Acad Sci U S A. 2011;108:5437-42.

[60] Zhu H, Pleil KE, Urban DJ, Moy SS, Kash TL, et al. Chemogenetic inactivation of ventral hippocampal glutamatergic neurons disrupts consolidation of contextual fear memory. Neuropsychopharmacology. 2014;39:1880-92.

[61] Packer AM, Roska B, Hausser M. Targeting neurons and photons for optogenetics. Nat Neurosci. 2013;16:805-15. 